\title{
Safety Wheel Chair for Paralysis Patient
}

\author{
Issa Saleh Ali Al-Sawaai \\ Vidhya Lavanya \\ Aniloy Frank
}

\author{
Middle East College \\ Middle East College \\ Middle East College
}

\begin{abstract}
This paper aims to develop a wheelchair for paralysis patients. The objectives of the paper include Fall detection, Forward bending detection, and SMS service. All these technologies have been covered in terms of their requirements, costs, system design, and system analysis. Fall detection is done using Accelerometer sensor. It detects the fall from the wheelchair of the patient who is having spinal problems. The sensor sends a signal to the controller and activates the buzzer. SMS will also be sent to the caretaker. The second technology used is forward bending detection. It is used for those who are bending their backs in order to inform them to sit properly. Ultrasonic sensor is used for this detection, alarm will be activated and SMS also will be sent. Next technology is the use of SMS service. If the patient is able to move one hand, he can use a keypad and 10 different buttons can be provided for different services. Depending on the keypad pressed, the corresponding SMS will reach the caretaker and the patient will be served accordingly. The proposed work is started after a thorough literature review. Arduino controller is used for getting the signal from the sensor and provide the information to the caretaker and switch ON buzzer. The proposed work is simulated using proteus and then implemented in hardware.
\end{abstract}

As an extension to the work, fire alarm system and automated wheel movement, live camera can also be added to improve the advantages of the safety wheel chair. The proposed work is funded by Research Council, Oman.

\section{Introduction}

The proposed work explained in the paper helps serving people with disabilities by developing several functions using the technologies. This work is mainly for the benefit of disabled people who use wheelchair. Three technologies are explained in this paper. Several research papers are discussed in the subsequent sections about the technologies used. This proposed work has first FALL DEDECTLON technology which work when the wheelchair with the person fall down. Then the alarm system in the chair will activate to attract people close to him and assist him immediately. Also, GSM module in the chair will send alert SMS to the person who is responsible of the disabled person. Using "FORWARD BEND DETECTION" is possible to find whether the person in the wheelchair is bending his back in front. In such a case, an alarm will be activated and SMS will be sent to the corresponding person.

The bending technology is for the patient who doesn't have the strength to bend their dorsum back again. The SMS service technology will also be useful when the patient presses a keypad which is kept near his hand. The keypad is of $4 * 3$ size and the messages will be sent for all the keypad from 0 to 6. The service can be extended for different other functions also (Diksha Goyal et al. 2013). The patient can now send SMS to his caretaker. Each button will provide unique message. This service is useful for the patient whose has one side paralysis. GSM module is attached with the prototype. Fixed text messages like "I'm hungry ", "I'm thirsty", "I need help" and so on can be used for the SMS service. The main objectives of the project are listed below.

- Using accelerator meter sensor to verify the angle of chair and send SMS, activate alarm if 


\section{Journal of Student Research}

not in straight position.

- Using ultrasonic sensor to find the position of the patient and if this sensor detects nothing activate alarm and send SMS.

- To send SMS if the person presses keys.

Methodology selection is important for obtaining the objectives in set timeline. Waterfall model is used for the proposed work. It has a linear sequential flow chart in which each process is flowing downward like waterfall. The current phase follows the previous phase. The major advantage of this model is that the timeline will be maintained and all the objectives are obtained in a sequential manner. Returning to the previous phase is not allowed in this model. Changing of requirements in the future phase is impossible. The reasons are listed below for the selection of the waterflow model obstacle (Chhaya. G. Patil, et al. 2014). The development is easy to structure and plan the work. The process is well defined and the objectives are obtained in timely manner. Returning to previous stage is costlier, and this project is less in cost. This work is done for enough duration of time.

Here are all comments that describe all stages on waterfall methodology (G. Găşpăresc et al. 2014)

- In the requirement stage, collection of materials and requirements needed for the project.

- In analysis stage, all the collected requirements will be verified with a thorough literature study.

- Next stage is design stage in which circuit design, system design, schematic diagram are finalized and analyzed.

- The above three stages are considered as planning stages and the real implementation is in the fourth stage. The connection of the circuit will be done once the simulation is done. The simulation of the work is done in tincerkad software.

- microcontroller is used after the work verification in simulation is done. Arduino is used, which is considered as the heart of the work. Programming is done and then downloaded in Arduino board which will follow the instructions of the written programming. The final product will be available and verified for all the objectives.

\section{Literature review}

Several literature papers are studied and as per the discussion (DW Hansen 2012) a wheelchair can be designed for the people who are disabled. It should have many facilities. The paper also explains the need of SMS service and the keypad usages and the gesture of the body. The paper explains the automation of the wheelchair for different gestures. The head and hand gestures are taken for considerations. Touchpad or keypad is used for hand gestures whereas accelerometer is used for head gestures (Prof. Vishal V. Pande, 2014). Switches are also used for hand gestures. The work is proposed with two modes of operation to connect all the needs of the user. The patient or the caretaker can switch from one mode to another (Zhmud V et al. 2015). A microcontroller is used to control the hand and head gesture variation from the switch and the accelerometer. Motors connected to the wheel will be responding to the control provided by the microcontroller, as per the gestures variation. (Rory A. Cooper, et al. 2000)

In the paper, Eyeball, Motion-Controlled, Wheelchair Using IR Sensor is designed to control wheelchair movement by the variation of the iris movement of the patient. This is useful for the old people and disabled patients who cannot talk. In this work, IR sensors are attached with the eye 
glass or eye frame. This IR sensor will follow up the movement of IRIS and the signals from the sensor will be transferred to the microcontroller. Microcontroller (pic18F452) is used to control the motors in the wheelchair. Since IR sensor will detect only the white objects, there is a necessity to make some unique sequence of bits for each movement direction of the iris eye. In the proposed work, all the IR sensors are fitted into left lens of the glasses. Through this technology, the wheelchair gets commands from a single eye. IR sensor will continuously transmit beam of IR rays. When a white rays come to the receiver, the rays will be reflected and captured, when black objects are in contact, the rays will be absorbed. The white object mentioned in the sclera and the iris will be the black object. The patient is educated to move the iris as per the requirement of the movement of the wheelchair. The movement is restricted to right and left directions controlled by the microcontroller (S.Shaheen, A.Umamakeswari, 2013).

Paralysis patients depend on other people and therefore need a wheelchair so they can move easily without any help (M. Reitbauer, 2008). The wheelchair should be designed in such a way that it is helpful for all the possible facilities. Ultrasound sensors can also be used for the wheelchair to avoid any obstacles in the path. 8051 microcontroller can be used to control ultrasound sensor, motor drivers and dc motors.

GH-311 ultrasound sensor is used to measure the distance to any object or obstacle (Chhaya. G. Patil, et al. 2014). The sensor will send the soundwave and will be reflected from the obstacle be received again by the sensor. The sensor sends the data to the microcontroller and will send the signal in terms of voltage to the motors connected to the wheelchair. The paper explains that the detection range in this sensor is $3 \mathrm{~cm}$ to $3 \mathrm{~m}$. This information from the literature paper is useful for connecting ultrasonic sensor to the wheelchair. (Bhagat Amar et al.2014).

The below section will be discussing the circuit design by providing block diagram, schematic diagram and analysis of the circuit.

\section{System block diagram}

System block diagram and flow of work will be helpful for finalizing the proposed work. System block diagram is shown in figure 1.

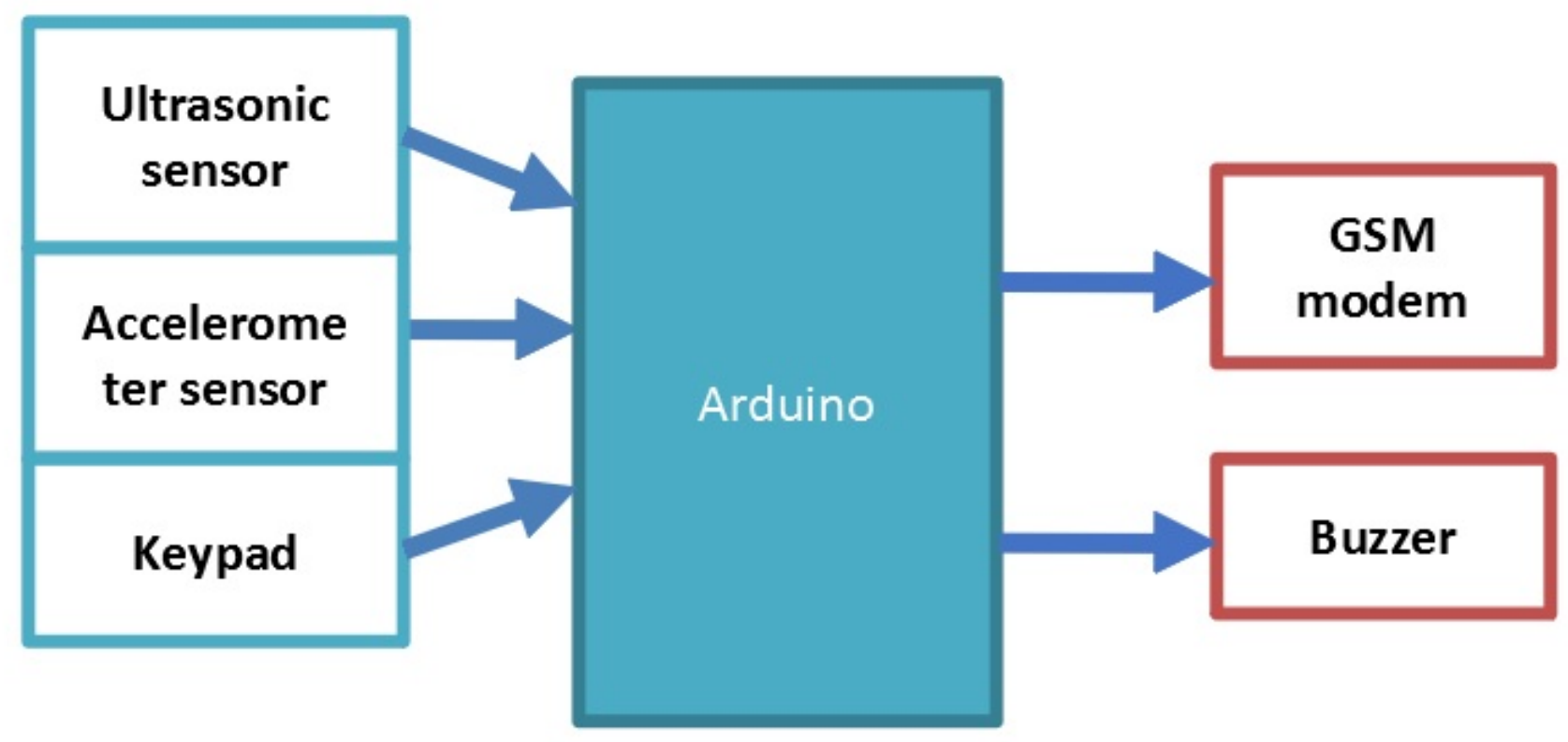

Figure 1. System Block diagram 
The proposed work consists of three technologies/ services; Fall detection, forward bed detection and SMS service. According to literature, ultrasonic sensor, keypad and accelerometer sensors are used for achieving the above services in a single wheelchair.

The flow graph of the work is explained below

1. If Accelerometer angle is not fixed, then the used service is fall detection. This represents the fall of the patient from the wheelchair and the alarm is activated. SMS is also sent to the caretaker.

2. In forward bend detection, if the push button is 1 then it means that the user is bending forward, the ultrasonic sensor value is also verified for this case. Alarm is also activated, and SMS is sent.

3. The third service is for the patient who is paralyzed from one side only. They can use keypad and provide information for the caretaker about their need by SMS. GSM modem is connected to the module.

\section{Fall detection technology}

ADXL335 Accelerometer is used as input. The changes in angles and rotation are sensed in all the three axes. This sensor is connected to the chair. Any variation in the accelerometer will be considered as falling case and the changes are informed to the microcontroller. The ADXL335 will send value of the fall axis as analog voltage between (0) to (5) volts to the analog inputs of Arduino. Figure 2 shows the implementation of fall detection service.

Figure 2. Fall detection circuit

\section{SMS services}

This keypad is used as an input. Several messages are stored in the microcontroller mapping with each keypad pressed. $4 * 4$ or $4 * 3$ keypads can be used and 16 or 12 messages can be saved. In this proposed work, keypads from 0 to 6 are used. The keypad is connected to 8 digital Arduino port pins.

The button value is decided by the row and column values and thus the pin is decided. Figure 3 explains the simulation circuit of the SMS service.

Figure 3. SMS services circuit

\section{Forward bending detection}

Ultrasonic sensor is used and it detects the position of the head in the chair. Pushbuttons are used to determine whether the person is sitting in chair or has fell down.

This equation 1 is used to calculate the distance of obstacles on ultrasonic. Figure 4 represents the bending detection circuit.

Figure 4. Forward bending detection circuit 


\section{System Simulation and Discussion}

The complete simulation of the project is done using the Proteus software. Once the connection of the circuit is finished, the code is downloaded from the IDE environment to the board which is in hex format. Figure 5 explains the complete system simulation.

Figure 5. System Simulation

\section{FALL DETECTION Simulation.}

Figure 6. GY-61 ADXL335 Simulation

Accelerometer (GY-61 ADXL335) is used to sense the curvature of the chair in cases of falling on the ground. The measuring directions are $\mathrm{z}, \mathrm{x}, \mathrm{y}$. The directions are measured and shown in figure 6 for different accelerometer. Since sensor cannot be used in the simulation, potential divider is used to show the different values connected to the pins for different ADXL values.

Ultrasonic sensor is fixed behind the chair of the patient to detect his back position. There is a pin called simPin in the sensor which shows the distance from the object. A potential divider circuit is again used to show the performance of the ultrasonic sensor.

\section{System Testing and Implementation}

All the three services are implemented and tested in the wheelchair. The final prototype of this circuit includes normal chair, plastic box to cover stripboard circuit and a piece of wood comes with large pushbutton as shown in figure 7

Figure 7. Implementation circuit

\section{Conclusion}

This proposed work aims to help disabled people with physical disabilities like paralysis, handicapped; those who are using the wheelchair in their daily lives. This work has three services and it can be universally used by the disabled and old people. The cost of the prototype is suitable, and the caretaker can get the information about the patients immediately. Literature reviews are done to finalize the objectives and selection of components. Suitable methodology selected to obtain the objectives. Datasheets of the sensors are used to fix the values of the Arduino control.

The work can be further extended to improve the services like increasing the SMS messages, and connecting IR sensor in the eye frame for the patients to freely move on their own.

\section{Acknowledgements}

The authors are thankful to Research council, Oman for funding this project.

\section{References}




\section{Journal of Student Research}

Fourth Middle East College Student Research Conference, Muscat, Sultanate of Oman

Bhagat Amar A., Doke Harshal R. ,Hajare Supriya D. , Prof. Rane D. B. (2014) “Multimode controlled wheelchair with safety precautions, International journal of emerging technology and advanced engineering" 4.

Chhaya. G. Patil, Sayali. K. Gharge, Sonal. V. Modhave, Y. S. Angal (2014), “Design of wheelchair using finger operation with image processing algorithms" International journal of research in engineering and technology,

Diksha Goyal and Dr. S.P.S. Saini (2013) “Accelerometer Based Hand Gesture Controlled Wheelchair", International Journal on Emerging Technologies 4(2): 15-20.

DW Hansen and P Majaranta (2012), "Basics of camera-based gaze tracking, Gaze interaction and applications of eye tracking: advances in assistive technologies", Medical Information Science Reference, Hershey, 21-26.

G. Găşpăresc and A. Gontean, (2014) "Performance evaluation of ultrasonic sensors accuracy in distance measurement." 11th International Symposium on Electronics and Telecommunications (ISETC), Timisoara, 1-4.

M. Reitbauer, (2008), “Keep an Eye on Information Processing: Eye Tracking Evidence for the Influence of Hypertext Structures on Navigational Behaviour and Textual Complexity, LSP and Professional Communication", 8.

Prof. Vishal V. Pande, (2014) "Hand Gesture Based Wheelchair Movement Control for Disabled Person Using MEMS" Int. Journal of Engineering Research and Applications, 4:152-158.

Rory A. Cooper, Jones, Shirley Fitzgerald, Michael L. Boninger, and Steven J. Albright (2000) "Analysis of Position and Isometric Joysticks for Powered Wheelchair Driving", IEEE transactions on biomedical engineering, 47: 12-15.

S.Shaheen, A.Umamakeswari,(2013) "Intelligent wheelchair for people with disabilities." International journal of engineering and technology, 5.

Zhmud V A, Trubin V G, Fiodorov D S, Ivoilov A Yu. (2015). "Development of Deflection Angle Stabilizing System for Balancing Robot." Journal of Advances in Management Sciences \& Information Systems 1: 65-82 